\title{
Gastrointestinal symptoms in patients with coronavirus disease 2019 (COVID-19) - friend or foe?
}

\author{
Konrad Lewandowski ${ }^{1}$, Magdalena Kaniewska ${ }^{1}$, Mariusz Rosołowski ${ }^{2,3}$, Adam Tworek ${ }^{1}$, Grażyna Rydzewska ${ }^{1,4}$ \\ ${ }^{1}$ Clinical Department of Internal Medicine and Gastroenterology with Inflammatory Bowel Disease Unit, Central Clinical \\ Hospital of the Ministry of Interior and Administration, Warsaw, Poland \\ ${ }^{2}$ Department of Internal Medicine and Hypertension, Medical University of Bialystok, Bialystok, Poland \\ ${ }^{3}$ Department of Hypertension, Gastroenterology and Internal Medicine, Medical University of Bialystok Clinical Hospital, \\ Bialystok, Poland \\ ${ }^{4}$ Collegium Medicum, Jan Kochanowski University, Kielce, Poland
}

Gastroenterology Rev 2022; 17 (3): 219-226

DOI: https://doi.org/10.5114/pg.2021.111000

Key words: coronavirus disease 2019 (COVID-19), gastrointestinal symptoms, coronavirus disease 2019 treatment.

Address for correspondence: Mariusz Rosołowski MD, PhD, Department of Internal Medicine and Hypertension, Medical University of Bialystok, 14 Zurawia St, 15-540 Białystok, Poland, phone: +48-85-8316506, e-mail: Mariusz.Rosolowski@umb.edu.pl

\begin{abstract}
Introduction: Gastrointestinal (GI) symptoms can be considered as a manifestation of coronavirus disease 2019 (COVID-19). Aim: Our study analysed GI symptoms depending on their occurrence, and their possible causes and impact on the course of COVID-19.

Material and methods: A retrospective, single-centre assessment of the frequency, risk factors, and impact of Gl symptoms in 441 patients with COVID-19.

Results: A statistically significant reduction in the length of stay (LOS) (15 days vs. 17 days; $p=0.04$ ), intensive care unit admission (ICU) $(16.9 \%$ vs. $26.8 \% ; p=0.02)$, and need for mechanical ventilation $(14.1 \%$ vs. $23.4 \% ; p=0.02)$ in the group who had experienced Gl symptoms before hospitalization was noticed. For comparison, patients who developed Gl symptoms during hospitalization had statistically significantly longer LOS (21 days vs. 15 days; $p=0.0001$ ), were more frequently admitted to the ICU ( $38.1 \%$ vs. $18.6 \%$; $p=0.0003)$, and had a higher need for mechanical ventilation $(32.7 \%$ vs. $16.2 \% ; p<0.001)$. Risk factors for GI symptoms during hospitalization in COVID-19 patients included age, Clostridioides difficile infection, and receiving certain treatment (antibiotics and lopinavir + ritonavir).

Conclusions: The Gl symptoms that developed before admission to hospital correlated with reduced severity of the course of COVID-19. However, in the group of patients who developed GI symptoms during hospitalization, attention should be paid to concomitant treatment. The use of antibiotics should be limited because they are associated with the deterioration of the course of COVID-19; one of the reasons might be changes in the intestinal microbiome.
\end{abstract}

\section{Introduction}

The most common symptoms of the coronavirus disease 2019 (COVID-19) are fever (83-99\%), cough (59-82\%), and fatigue (44-70\%) [1-3]. However, gastrointestinal (Gl) symptoms such as nausea, vomiting, diarrhoea, lack of appetite, and abdominal pain are also important features of COVID-19 [4, 5]. The most common is diarrhoea (13\%), followed by nausea or vomiting (10\%), and abdominal pain [6, 7].

There are many theories regarding the pathogenesis of GI symptoms during COVID-19, which include: affinity of SARS-CoV-2 for the angiotensin-converting enzyme 2 (ACE2) receptor, disturbances in the intestinal microbiome, the theory of cytokine storm, and drug-related complications.

The relationship with the presentation of GI symptoms can be explained by the fact that SARS-CoV-2 has a high affinity for the ACE2 receptor (a significant amount of which is found in the cells of the gastrointestinal tract), and perhaps that is why the genetic material of the virus is present in the stool of COVID-19 patients [7-9]. It was found that the genetic material of SARS-CoV-2 present in the cells of the gastrointestinal epithelium causes impairment of its function or even destruction, which may manifest itself in Gl symptoms, 
such as diarrhoea [10]. Gl symptoms also occurred in the course of the acute respiratory syndrome coronavirus (SARS-CoV) and the Middle East respiratory syndrome coronavirus (MERS-CoV) [11-13]. Finally, the presence of SARS-CoV-2 in human enterocytes was confirmed in in-vitro studies, which seems to confirm the above hypothesis [14].

A particular association between GI symptoms with high levels of Interleukin (IL) 6 (IL-6), IL-8, and $\mathrm{IL}-10$ has been reported in patients with poor prognosis, who were admitted to the ICU due to respiratory failure [15-18]. The significantly increased concentration of pro-inflammatory cytokines such as IL-1, IL-6, IL-18, tumor necrosis factor- $\alpha$ (TNF- $\alpha$ ), and interferon, which takes place in the "cytokine storm", causes the mobilization of some cells of the immune system. The action of neutrophils, macrophages, and T lymphocytes results in the destruction of the vascular barrier and hence the destruction of the epithelium in the gastrointestinal tract and other organs such as the lungs. Because of time, the whole process leads to multi-organ failure.

Another hypothesis of the occurrence of Gl symptoms concerns disturbances of the gut microbiome. In the work of Zuo et al. COVID-19 patients showed significant variations in the composition of the gut microbiome, characterized by an increase in opportunistic pathogens and a decrease in the number of beneficial commensals during hospitalization. This probably contributes to an increase in the permeability of the intestinal barrier, generates inflammation, and leads to damage to the epithelium in the gastrointestinal tract, which causes Gl symptoms such as abdominal pain and diarrhoea. The number of Coprobacillus, Clostridium ramosum, and Clostridium hathewayi at the beginning of hospitalization correlated with the severity of COVID-19. On the other hand, Alistipes onderdonkii, Faecalibacterium prausnitzii, Bacteroides dorei, Bacteroides thetaiotaomicron, Bacteroides massiliensis, and Bacteroides ovatus were the main bacterial species showing a negative correlation with the intensity of COVID-19. In addition, downregulation of ACE2 expression in the intestine of mice showed a significant inverse correlation with the faecal SARS-CoV-2 viraemia in COVID-19 patients [19].

There is no specific antiviral treatment recommended for COVID-19. Therapy is mainly symptomatic, and oxygen is the first step in managing respiratory distress. During the first wave of the pandemic, chloroquine, hydroxychloroquine, and lopinavir + ritonavir were used in COVID-19 treatment. Inflammation inhibitors like tocilizumab, bamlanivimab + etesevimab, casirivimab + imsevimab also appear to be effective [20]. The use of glucocorticosteroids and anticoagulants gained impor- tance after publication of the RECOVERY trial [21, 22]. Moreover, the treatment of bacterial coinfections in the course of COVID-19 involves antibiotics, which were often used in over $70 \%$ of patients [23]. The extensive use of antibiotics is a risk factor for the occurrence of Clostridioides difficile infection (CDI) [24]. Due to the high use of antibiotics in COVID-19, an increased incidence of CDI has been observed [25].

The impact of Gl symptoms in COVID-19 patients on their prognosis is not clearly defined. Currently available data are basically contradictory; GI symptoms may correlate with deterioration or improved patient's prognosis [26-28]. There are no studies available that analyse the prognosis of COVID-19 patients and factors depending on the time of Gl symptoms.

On 16 March 2020, the Central Clinical Hospital of the Ministry of Interior and Administration in Warsaw was transformed into a place only for the care of patients with COVID-19. As many as $57.8 \%$ of patients presented with GI symptoms, which was an extremely large number compared to other studies. Due to their frequent occurrence, we decided to perform an analysis of those symptoms depending on the time of their presentation, i.e. before admission to hospital and during hospitalization.

\section{Aim}

We analysed retrospectively the frequency of gastrointestinal symptoms in the group that presented with these symptoms before admission and during the stay. Both groups were screened for the course of the disease, including length of stay (LOS), need for intensive care unit (ICU) admission, need for mechanical ventilation, and mortality. We analysed potential risk factors for their occurrence, such as drugs used during COVID-19 or CDI.

\section{Material and methods}

We conducted a retrospective analysis of risk factors and the impact of gastrointestinal symptoms on a cohort of 441 patients with confirmed SARS-CoV-2 infection, who were hospitalized between 16 March and 15 June 2020 at the Central Clinical Hospital of the Ministry of the Interior and Administration in Warsaw. Patients enrolled in the study were 18 years of age or older. The cohort was divided into 2 groups: patients who had developed GI symptoms before admission and patients who developed Gl symptoms during hospitalization. GI symptoms were recorded based on patients' interviews. Glucocorticosteroid use was not analysed because it was introduced into guidelines on the treatment of COVID-19 in Poland after the studied period. 
We included all hospitalized patients over the aforementioned period; no patient was excluded from the study.

The main purpose of the article was to assess the incidence, risk factors, and effect of Gl symptoms on the course of COVID-19 depending on the time of symptom onset (before and during hospitalization).

\section{Statistical analysis}

Statistical analysis was conducted with the use of Statistica software, version 13.0 (https://www.statsoft. $\mathrm{pl} /$ ). Nominal variables were presented as $n$ (\% frequency of group), while continuous variables as mean (SD) or median (Q1; Q3), depending on the normality of data distribution. Data normality was verified using the Shapiro-Wilk test and based on visual assessment of histograms. Groups were compared with $\chi^{2}$ test for dichotomous variables and with $t$-test or Mann-Whitney $U$ test for continuous variables, as appropriate. All tests were 2 -sided, with $p=0.05$ considered significant.

\section{Bioethical considerations}

The study protocol was approved by the Bioethics Committee of the Central Clinical Hospital of the Ministry of the Interior and Administration in Warsaw. The researchers analysed anonymized data.

\section{Results}

A total of 441 hospitalized patients with COVID-19 were included. Among them, 255 (57.8\%) presented
Table I. Prevalence of individual GI symptoms in the COVID-19 patients (cases); general population $(n=441)$

\begin{tabular}{lc} 
Parameter & $\boldsymbol{N}(\%)$ \\
\hline Gastrointestinal symptoms: & $255(57.8)$ \\
\hline Abdominal pain & $95(37.3)$ \\
\hline Diarrhoea & $109(42.7)$ \\
\hline Nausea & $32(12.5)$ \\
\hline Vomiting & $37(14.5)$ \\
\hline Lack of appetite & $124(48.6)$ \\
\hline No gastrointestinal symptoms & $186(42.2)$
\end{tabular}

Gl symptoms. The most common symptom was lack of appetite, which was reported in 124 (48.6\%) cases. Other Gl symptoms like diarrhoea were observed in 109 (42.7\%), abdominal pain in 95 (37.3\%), vomiting in $37(14.5 \%)$, and nausea in 32 (12.5\%) cases. The distribution of sex was similar in both groups: $49.4 \% \mathrm{fe}-$ males in the group with Gl symptoms (cases) vs. $48.4 \%$ in the group without $\mathrm{Gl}$ symptoms (controls); $p=0.083$. Patients were significantly older: 68.44 years vs. 64.45 years in controls; $p=0.02$ and had significantly more comorbidities: $91.0 \%$ vs. $82.3 \% ; p=0.007$, respectively (Tables I and II).

The cohort was divided into 2 groups: 142 (32.2\%) patients who had developed Gl symptoms before admission and 113 (25.62\%) patients who developed GI symptoms during hospitalization (Table III).

In the group of 142 (32.2\%) patients who had developed Gl symptoms prior to admission to hospital vs.

Table II. Study cohort, presence of GI symptoms, and risk analysis

\begin{tabular}{|c|c|c|c|c|}
\hline Characteristics & $\begin{array}{c}\text { All } \\
(n=441)\end{array}$ & $\begin{array}{c}\text { No abdominal } \\
\text { symptoms } \\
(n=186)\end{array}$ & $\begin{array}{l}\text { Abdominal } \\
\text { symptoms } \\
(n=255)\end{array}$ & $P$-value \\
\hline Sex, female, $n(\%)$ & $216(49.0)$ & $90(48.4)$ & $126(49.4)$ & 0.83 \\
\hline Age [years] mean (SD) & $66.76(18.4)$ & $64.45(20.48)$ & $68.44(16.62)$ & 0.02 \\
\hline Hospitalization [days] median (Q1; Q3) & $18(10.00 ; 24.00)$ & $15.00(9.00 ; 22.00)$ & $17.00(10.00 ; 25.00)$ & 0.1 \\
\hline Comorbid diseases, $n(\%)$ & $385(87.3)$ & $153(82.3)$ & $232(91.0)$ & 0.007 \\
\hline Need for mechanical ventilation, $n(\%)$ & $90(20.4)$ & $33(17.7)$ & $57(22.4)$ & 0.24 \\
\hline Stay in the ICU, $n(\%)$ & $104(23.6)$ & $37(19.9)$ & $67(26.3)$ & 0.11 \\
\hline Mortality, $n(\%)$ & $148(33.6)$ & $49(26.3)$ & $99(38.8)$ & 0.006 \\
\hline CDI, $n(\%)$ & $48(10.9)$ & $6(3.2)$ & $42(16.5)$ & $<0.001$ \\
\hline PPI use, $n(\%)$ & $197(44.7)$ & $66(35.5)$ & $131(51.3)$ & $<0.001$ \\
\hline Any antibiotic used, $n(\%)$ & $354(80.3)$ & $133(71.5)$ & $221(86.7)$ & $<0.001$ \\
\hline Azithromycin use, $n(\%)$ & $214(48.5)$ & $83(44.6)$ & $131(51.4)$ & 0.16 \\
\hline Other antibiotic than azithromycin used, $n(\%)$ & $300(68.0)$ & $111(59.7)$ & $189(74.1)$ & 0.001 \\
\hline Chloroquine use, $n$ (\%) & $321(72.8)$ & $132(71.0)$ & $189(74.1)$ & 0.46 \\
\hline Lopinavir and ritonavir use, $n(\%)$ & $60(13.6)$ & $19(10.2)$ & $41(9.3)$ & 0.08 \\
\hline
\end{tabular}


Table III. Onset of abdominal symptoms before hospitalizations vs. no abdominal symptoms

\begin{tabular}{|c|c|c|c|c|}
\hline Characteristic & $\begin{array}{c}\text { All } \\
(n=441)\end{array}$ & $\begin{array}{l}\text { Start of abdominal } \\
\text { symptoms before } \\
\text { hospitalization } \\
(n=142)\end{array}$ & $\begin{array}{l}\text { No abdominal } \\
\text { symptoms before } \\
\text { hospitalization } \\
(n=299)\end{array}$ & $P$-value \\
\hline Sex, female, $n(\%)$ & $216(49.0)$ & $69(48.6)$ & $147(49.2)$ & 0.91 \\
\hline Age [years] mean (SD) & $66.76(18.4)$ & $67.13(17.6)$ & $66.58(18.84)$ & 0.77 \\
\hline Comorbid disease, $n(\%)$ & $385(87.3)$ & $129(90.8)$ & $256(85.6)$ & 0.12 \\
\hline Hospitalization [days] median (Q1; Q3) & $18(10.00 ; 24.00)$ & $15.00(10: 00 ; 20.00)$ & $17.00(10.00 ; 25.00)$ & 0.04 \\
\hline ICU stay, $n(\%)$ & $104(23.6)$ & $24(16.9)$ & $80(26.8)$ & 0.02 \\
\hline Need for mechanical ventilation, $n(\%)$ & $90(20.4)$ & $20(14.1)$ & $70(23.4)$ & 0.02 \\
\hline Mortality, $n(\%)$ & $148(33.6)$ & $53(37.3)$ & $95(31.8)$ & 0.24 \\
\hline $\mathrm{CDI}, n(\%)$ & 48 (10.9) & $17(12.0)$ & $31(10.4)$ & 0.61 \\
\hline PPI use, $n(\%)$ & $197(44.7)$ & $72(50.7)$ & $125(41.8)$ & 0.08 \\
\hline
\end{tabular}

Table IV. Group of patients with GI symptoms before admission to hospital vs. no Gl symptoms; general population $(n=441)$

\begin{tabular}{lc} 
Parameter & $\boldsymbol{N}(\%)$ \\
\hline Gastrointestinal symptoms before admission: & $151(34.2)$ \\
\hline Abdominal pain & $70(15.9)$ \\
\hline Diarrhoea & $60(13.6)$ \\
\hline Nausea & $10(2.2)$ \\
\hline Vomiting & $11(2.5)$ \\
\hline Lack of appetite & $84(19.9)$ \\
\hline No gastrointestinal symptoms before admission & $290(65.8)$
\end{tabular}

patients without Gl symptoms, we observed a statistically significant reduction in the LOS: 15 days vs. 17 days; $p=0.04$, a reduction in the frequency of stays in the ICU: $16.9 \%$ vs. $26.8 \% ; p=0.02$, and a lower need for mechanical ventilation: $14.1 \%$ vs. $23.4 \% ; p=0.02$, respectively. No statistical significance was noted for mortality among patients with GI symptoms prior to admission to hospital, i.e. 53 (37.3\%) vs. asymptomatic groups, i.e. 95 (31.8\%); $p=0.24$. Sex distribution, age, comorbid diseases, proton pump inhibitors (PPI) used, and frequency of CDI were not risk factors for the presentation of Gl symptoms before hospitalization ( $\mathrm{Ta}$ ble III). In the group of patients with GI symptoms before admission to hospital, the most common symptom was lack of appetite in 84 (19.9\%), followed by abdominal pain in $70(15.9 \%)$, diarrhoea in 60 (13.6\%), vomiting in 11 (2.5\%), and nausea in 10 (2.2\%) cases (Table IV).

As many as 113 (25.62\%) patients who developed Gl symptoms during their hospital stay (vs. patients without Gl symptoms) had statistically significantly longer LOS, i.e. 21 days vs. 15 days; $p=0.0001$, more frequent ICU stays, i.e. $38.1 \%$ vs. $18.6 \% ; p=0.0003$, a higher need for mechanical ventilation, i.e. $32.7 \%$ vs. $16.2 \% ; p<0.001$, and a more frequent incidence of CDI, i.e. $22.1 \%$ vs. $7.0 \%$; $p=0.0001$, respectively. No statistical significance was noted for mortality among patients with Gl symptoms during their stay vs. asymptomatic groups, i.e. 46 (40.1\%) vs. 102 (31.1\%), respectively; $p=0.06$. Age, frequency of CDI, and some specific drugs for COVID-19 treatment (any antibiotics, azithromycin, antibiotics other than azithromycin and lopinavir + ritonavir) were risk factors for the presentation of $\mathrm{Gl}$ symptoms during hospitalization. Sex, comorbid diseases, PPI, and chloroquine use were not risk factors for presenting with $\mathrm{Gl}$ symptoms during hospitalization (Table V). In the group of patients with Gl symptoms during hospitalization, the most common was diarrhoea, present in 49 (11.1\%) cases, followed by lack of appetite in 40 (9.1\%), vomiting in 26 (5.9\%), abdominal pain in 25 (5.7\%), and nausea in 22 (5.0\%) (Table VI).

\section{Discussion}

As a COVID-dedicated hospital with the highest level of reference, we dealt with a group of patients who were in advanced age and had many comorbidities. In the other analysed studies, the cohorts were younger, which makes our analysis much more difficult, because advanced age is an independent deteriorating prognostic factor in COVID-19 [28]. The mean age of our group of patients was 66.76 years, compared to the mean age in the study by Cheung et al. at 45.1 years, Livanos et al. at 60.5 years, and Pan et al. at 52.9 years $[6,28,29]$.

Another unfavourable prognostic factor in COVID-19 is the presence of comorbidities [30, 31]. In our study, over $87.3 \%$ of patients had at least 1 comorbid disease, which is quite typical for elderly patients. In more than half of the younger cohort from the study of Cheung et al., comorbidities were not analysed, which could be 
Table V. Onset of abdominal symptoms during hospitalizations vs. no abdominal symptoms

\begin{tabular}{lcccc} 
Characteristics & $\begin{array}{c}\text { All } \\
(n=441)\end{array}$ & $\begin{array}{c}\text { Start of abdominal } \\
\text { symptoms during } \\
\text { hospitalization } \\
(n=113)\end{array}$ & $\begin{array}{c}\text { No abdominal } \\
\text { symptoms during } \\
\text { hospitalization } \\
(n=328)\end{array}$ & $P$-value \\
\hline Sex, female, $n$ (\%) & $216(49.0)$ & $57(50.4)$ & $159(48.5)$ & 0.72 \\
\hline Age [years] mean (SD) & $66.76(18.4)$ & $70(15.25)$ & $65.61(19.3)$ & 0.03 \\
\hline Hospitalization [days] median (Q1; Q3) & $18(10.00 ; 24.00)$ & $21.00(10.0 ; 32.00)$ & $15.00(9.00 ; 21.25)$ & 0.0001 \\
\hline Need for mechanical ventilation, $n(\%)$ & $90(20.4)$ & $37(32.7)$ & $53(16.2)$ & $<0.001$ \\
\hline ICU stay, $n$ (\%) & $104(23.6)$ & $43(38,1)$ & $61(18.6)$ & 0.0003 \\
\hline Mortality, $n(\%)$ & $148(33.6)$ & $46(40.1)$ & $102(31.1)$ & 0.06 \\
\hline CDI, $n$ (\%) & $48(10.9)$ & $25(22.1)$ & $23(7.0)$ & 0.0001 \\
\hline Comorbid disease, $n(\%)$ & $385(87.3)$ & $103(91.2)$ & $282(86.0)$ & 0.15 \\
\hline PPI use, $n$ (\%) & $197(44.7)$ & $59(52.2)$ & $138(42.1)$ & 0.06 \\
\hline Any antibiotic used, $n(\%)$ & $354(80.3)$ & $104(92.0)$ & $250(76.2)$ & 0.0002 \\
\hline Azithromycin use, $n(\%)$ & $214(48.5)$ & $68(60.2)$ & $146(44.5)$ & 0.004 \\
\hline Other antibiotic used, $n(\%)$ & $300(68.0)$ & $92(81.4)$ & $208(63.4)$ & 0.0004 \\
\hline Chloroquine, $n(\%)$ & $321(72.8)$ & $90(79.6)$ & $231(70.4)$ & 0.06 \\
\hline Lopinavir and ritonavir, $n(\%)$ & $60(13.6)$ & $24(21.2)$ & $36(11.0)$ & 0.006
\end{tabular}

due to the fact that patients of such a young age were not burdened with typical chronic diseases, e.g. diseases of the cardiovascular and respiratory systems. In other analysed studies, the number of comorbidities was clearly lower. In the study by Livanos et al., only $16.3 \%$ of patients had comorbidities, and in the study by Pan et al. it was almost half $(49.99 \%)$ of the cohort $[6,28,29]$.

Lack of appetite was the most common GI symptom in our study, which occurred in 124 (48.6\%) cases. As a highly non-specific symptom, it was not analysed in the study by Livanos et al. or Pan et al. Cheung et al. also found that anorexia was the most common symptom in the cohort with GI symptoms. It was present in $26.8 \%$, which is almost half the frequency found in our cohort. The evidently greater lack of appetite in our cohort could be associated with a significantly higher age of patients and a greater number of comorbidities $[6,28,29]$. These factors, along with severe COVID-19 course that required hospitalization, could reduce appetite, especially considering the mechanism of inflammation and the so-called "cytokine storm", with a high number of inflammatory mediators inversely correlating with appetite [31].

Diarrhoea, which is defined as more than 3 bowel movements a day, was also a common symptom among COVID-19 patients. Our study found it in $42.7 \%$ of patients. A much lower rate of diarrhoea in the Cheun et al. study, i.e. $12.5 \%$ of patients, may suggest that the symptom was overlooked in the early stages of the pandemic. When the battle against COVID-19 began,
Table VI. Group of patients with Gl symptoms during hospitalization vs. no Gl symptoms; general population $(n=441)$

\begin{tabular}{lc} 
Parameter & $\boldsymbol{N}(\%)$ \\
\hline $\begin{array}{l}\text { Gastrointestinal symptoms during } \\
\text { hospitalization }\end{array}$ & $162(36.7)$ \\
\hline Abdominal pain & $25(5.7)$ \\
\hline Diarrhoea & $49(11.1)$ \\
\hline Nausea & $22(5.0)$ \\
\hline Vomiting & $26(5.9)$ \\
\hline Lack of appetite & $40(9.1)$ \\
\hline $\begin{array}{l}\text { No gastrointestinal symptoms before } \\
\text { admission }\end{array}$ & $279(63.3)$
\end{tabular}

doctors focused primarily on fighting acute respiratory failure. This was also cited as a limitation in this work. A similar number of patients with diarrhoea was reported on by Livanos et al. (39\%) and Pan et al. (34\%), where the cohort patients were of relatively similar age and had fewer comorbidities [6, 28, 29]. The occurrence of diarrhoea in patients with COVID-19 may be related to the use of the ACE2 receptor by SARS-CoV-2. The entry of SARS-CoV-2 into the host cell is mediated by the interaction between the encapsulated viral spike protein and the host receptor, consisting of ACE2. Mouse models show that the presence of ACE2 alterations is associated with colitis, suggesting that virus activity may cause enzyme modifications, increasing the susceptibility to intestinal inflammation and diarrhoea [32]. 
Moreover, the aspect of disturbing the intestinal microbiota in the formation of diarrhoea is emphasized. In the publication of Zuo et al., patients with SARS-CoV-2 infection were found to have significant changes in their gut microbiome compared with controls, characterized by enrichment of opportunistic pathogens and depletion of beneficial commensals [19].

Abdominal pain, defined as a non-specific subjective symptom characterized by pain localized in the abdominal cavity, occurred in $37.3 \%$ of patients in our study. In a study by Livanos et al. abdominal pain was not reported. This symptom was a rare occurrence in the study by Pan et al., being present in $1.9 \%$ of patients, but was more frequent, i.e. present in $9.2 \%$ of patients, in the study by Cheung et al. $[6,28,29]$.

Vomiting and nausea are other important, although non-specific gastrointestinal symptoms. In our study, vomiting occurred in $14.5 \%$ and nausea in $12.5 \%$ of cases. A similar rate of nausea and vomiting, i.e. $10.2 \%$, was found in the study by Cheung et al. In the study by Pan et al. only vomiting was analysed, with the rate of $3.9 \%$, which was relatively small. A similar rate of vomiting was found in the study by Livanos et al., i.e. 13\%, while nausea was found in more than $25 \%$ [6, 28, 29].

The results of our study on the occurrence of Gl symptoms before hospitalization confirm the conclusions of the study by Livanos et al. because it is evident that the presence of GI symptoms before hospitalization correlates with improved course of the disease, particularly shown by shorter LOS, and reduced need for mechanical ventilation or stay in the ICU. In the study by Livanos et al., $47 \%$ of patients reported GI symptoms. In our study, GI symptoms occurred much more often, i.e. in $57.8 \%$ of the entire cohort. The researchers did not take into consideration symptoms such as abdominal pain and lack of appetite, which may have contributed to the lower incidence of Gl symptoms. The presence of diarrhoea (39\% vs. $42.7 \%$, respectively) and vomiting ( $13 \%$ vs. $12.5 \%$, respectively) was similar. There was a difference in the incidence of nausea (25\% vs. $12.5 \%$, respectively); in the study by Livanos et al., it occurred twice as often. The group presenting the Gl symptoms was younger than ours, i.e. 61 years vs. 68.44 years, respectively. The biggest difference between the cohorts was in the distribution of comorbidities. In the study by Livanos et al. only $16.3 \%$ of patients with Gl symptoms had comorbidities, as compared to $91 \%$ of patients in our study. It is worth emphasizing that the Central Clinical Hospital of the Ministry of the Interior and Administration in Warsaw had the highest referential degree, so it hospitalized the most difficult cases with the highest number of comorbidities, which required the most specialized care. In the study by Livanos et al., the group with Gl symptoms had a significantly lower mortality as compared to the group from our study, i.e. $15.7 \%$ vs. $38.8 \%$, respectively. The authors concluded that Gl symptoms remain significantly correlated with better COVID-19 outcomes. The lower mortality in their study certainly resulted from the younger age of the patients, and above all from the fact that they had significantly fewer comorbidities. An external validation cohort further confirmed decreased mortality in COVID-19 patients with Gl symptoms. In the second validation cohort, the presence of Gl symptoms was used to predict reduced disease severity and mortality. During short-term follow-up there was a significantly lower rate of death. The inclusion of 3 independent cohorts totalling 1163 patients enhanced the strength and validity of their findings. Furthermore, their model to predict COVID-19 severity and mortality was improved by the inclusion of Gl symptoms suggesting that intestinal parameters should be considered in initial assessments and stratification of COVID-19 patients. These data suggest the potential for tissue-specific response to SARS-CoV-2 and the potential for attenuation of viral pathogenicity by the GI tract. Compared to our study, the 3 cohorts from the Livanos et al. study were younger and had fewer comorbidities, which contributed to a significant reduction in the severity of the disease, and thus mortality. It was also emphasized that the attenuation of SARS-CoV-2 by the GI tract could significantly improve the disease course and reduce mortality. The findings from our study are similar for the group who presented with GI symptoms before admission to hospital [28].

On the other hand, our results concerning the occurrence of $\mathrm{Gl}$ symptoms during hospitalization show an evidently worse course of the disease, defined by prolonged LOS, and increased need for mechanical ventilation and for ICU stay. The presence of those GI symptoms significantly correlates with some drugs used during COVID-19 treatment (antibiotics and lopinavir + ritonavir) and with the frequency of CDI, which is significantly associated with the widespread use of antibiotics [24, 25]

In the study by Pan et al., 204 patients with COVID-19 from 3 hospitals in Hubei were enrolled. The mean age was 52.9 years, which is much lower than in our cohort ( 68.44 years). As many as $50.5 \%$ of their patients had Gl symptoms, vs. $57.8 \%$ according to our results. Lack of appetite occurred in $78.6 \%$ of cases, which was higher than in our cohort (48.6\%). Diarrhoea was reported less frequently than in our study, i.e. in $34 \%$ vs. $42.7 \%$ of cases, respectively. Abdominal pain (1.9\% vs. $37.3 \%$, respectively) and vomiting (3.9\% vs. $14.5 \%$, respectively) were reported less frequently as compared to our study. The authors excluded the lack of 
appetite in analysis, because according to them it was not specific for the GI tract. There were $18.6 \%$ of cases with $\mathrm{Gl}$ symptoms after the lack of appetite was excluded. As compared to our study, the lack of appetite was also the most common symptom. Its exclusion seems justified because the reason for its occurrence may be related to the "cytokine storm", patient's age, or number of comorbidities. In their study, Pan et al. analysed contact history, comorbidities (respiratory, digestive, cardiovascular, nervous, endocrine system diseases, and malignant tumour), medical treatment after admission (antibiotic, antifungal, antiviral treatment, glucocorticosteroids, nebulized interferon, and intravenous immunoglobulin), and clinical outcome (duration of hospitalization, stay in the ICU, discharge from hospital, or death). The study group was younger compared to our cohort and had fewer comorbidities. No statistical significance was demonstrated in the Gl symptoms group for comorbidities, medical treatment, or clinical outcome. In our study, the outcome of COVID-19 infection varied in both groups, and significant differences were noted in LOS, stay in the ICU, and need for mechanical ventilation. Mortality was higher in our study (38.8\%) as compared to the study by Pan et al. (18.45\%), which could be related to the lower age of their cohort and fewer comorbidities. There was more widespread antibiotic treatment in our group than in the Pan et al. study, but they did not analyse the incidence of CDI due to the high rate of antibiotic use [29].

In the meta-analysis by Cheung et al., among 4243 patients with COVID-19 from 6 countries, all GI symptoms (including loss of appetite, nausea and vomiting, diarrhoea, or abdominal pain) were observed in $17.6 \%$ of patients, which is much less when compared to our results. Loss of appetite was the most common Gl symptom (26.8\%), followed by diarrhoea (12.5\%), nausea and vomiting (10.2\%), and abdominal pain or discomfort (9.2\%). The Gl symptoms may have been overlooked at the beginning of the outbreak, especially in the study from Wuhan, because China was the first country to develop COVID-19, and this may have contributed to underestimated Gl symptoms in previous studies. Cheung et al. concluded that patients with Gl symptoms had a more severe disease course compared to the asymptomatic group ( $17.1 \%$ vs. $11.8 \%$, respectively). In our study, there were $57.8 \%$ of Gl symptoms compared to $17.6 \%$ in the meta-analysis by Cheung, who admits in his study's limitations that these symptoms may have been underreported in some studies, which may lead to a lower pooled prevalence rate [6].

The strength of our study is a cohort treated in a uniform way from one centre and one geographical region. Another important feature is the analysis of drugs used during hospitalization based on electronic patient charts. Our study has also several limitations. First, the study is retrospective in nature, and risk factors for the severe/critical type of COVID-19 were identified according to patients' data on admission. Second, we did not look for SARS-CoV-2 RNA in stool samples, despite over $50 \%$ of viral RNA being detected in the faeces according to another study. Third, we analysed only hospitalized patients; therefore, mostly patients with severe COVID-19 were included in the analysis.

The data published until now on the presentation of GI symptoms in COVID-19 are very interesting. There are many discrepancies concerning their impact on the course of the disease, with most studies showing a negative impact on COVID-19 course and mortality. So far, these studies have not analysed other possible factors related to GI symptoms. Our work confirms GI symptoms as a manifestation of COVID-19. We analysed GI symptoms depending on the time of their onset and tried to elucidate risk factors for the development of $\mathrm{Gl}$ symptoms during hospitalization. It turned out that antibiotics and lopinavir-ritonavir significantly correlated with symptoms during hospitalization and a worsened course of the disease. We understand that correlation does not mean causation, but we think that the fact that the time of occurrence of GI symptoms correlates with COVID-19 severity requires further research on the mechanisms behind it and can be useful for patient stratification. The fact that certain medications correlate with Gl symptoms during hospitalization is also thought- and hypothesis-provoking and requires further elucidation.

\section{Conclusions}

The Gl symptoms that developed prior to admission to hospital correlate with a reduced severity of the disease course; conversely, Gl symptoms that develop during hospitalization correlate with a more sever COVID-19 course. Certainly, more in-depth research is required. However, inclusion of Gl symptoms (before and during hospitalization) in disease severity prediction models seems promising. On the other hand, in the group of patients who develop Gl symptoms during their stay, attention should be paid to concomitant medication.

\section{Conflict of interest}

The authors declare no conflict of interest.

\section{References}

1. Huang C, Wang Y, Li X, et al. Clinical features of patients infected with 2019 novel coronavirus in Wuhan, China. Lancet 2020; 395: 497-506. 
2. Chen N, Zhou M, Dong X, et al. Epidemiological and clinica characteristics of 99 cases of 2019 novel coronavirus pneumonia in Wuhan, China: a descriptive study. Lancet 2020; 395 507-13.

3. Guan WJ, Zhong NS. Clinical characteristics of Covid-19 in China. Reply. N Engl J Med 2020; 382: 1861-62.

4. Ramachandran P, Onukogu I, Ghanta S, et al. Gastrointestinal symptoms and outcomes in hospitalized coronavirus disease 2019 patients. Dig Dis 2020; 38: 373-9.

5. Rokkas T. Gastrointestinal involvement in COVID-19: a systematic review and meta-analysis. Ann Gastroenterol 2020; 33: 355-65.

6. Cheung KS, Hung IFN, Chan PPY, et al. Gastrointestinal manifestations of SARS-CoV-2 infection and virus load in fecal samples from a Hong Kong Cohort: systematic review and meta-analysis. Gastroenterology 2020; 159: 81-95.

7. Young BE, Ong SWX, Kalimuddin S, et al. Epidemiologic features and clinical course of patients infected with SARS-CoV-2 in Singapore. JAMA 2020; 323: 1488-94.

8. Cheung KS, Hung IFN, Leung WK. Association between famotidine use and COVID-19 severity in Hong Kong: a territory-wide study. Gastroenterology 2021; 160: 1898-9.

9. Wu Y, Ho W, Huang Y, et al. SARS-CoV-2 is an appropriate name for the new coronavirus. Lancet 2020; 395: 949-50.

10. Xiao F, Tang M, Zheng X, et al. Evidence for gastrointestinal infection of SARS-CoV-2. Gastroenterology 2020; 158: 1831 $3 e 1833$.

11. Leung WK, To KF, Chan PK, et al. Enteric involvement of severe acute respiratory syndrome-associated coronavirus infection. Gastroenterology 2003; 125: 1011-7.

12. Chan KH, Poon LL, Cheng VC, et al. Detection of SARS coronavirus in patients with suspected SARS. Emerg Infect Dis 2004, 10: 294-9.

13. Cotten M, Watson SJ, Kellam P, et al. Transmission and evolution of the Middle East respiratory syndrome coronavirus in Saudi Arabia: a descriptive genomic study. Lancet 2013; 382: 1993-2002.

14. Lamers MM, Beumer J, van der Vaart J, et al. SARS-CoV-2 productively infects human gut enterocytes. Science 2020; 369 $50-4$

15. Xu X, Yu MQ, Shen Q, et al. Analysis of inflammatory parameters and disease severity for 88 hospitalized COVID-19 patients in Wuhan, China. Int J Med Sci 2020; 17: 2052-62.

16. Akbari $\mathrm{H}$, Tabrizi R, Lankarani KB, et al. The role of cytokine profile and lymphocyte subsets in the severity of coronavirus disease 2019 (COVID-19): a systematic review and meta-analysis. Life Sci 2020; 258: 118167.

17. Del Valle DM, Kim-Schulze S, Huang HH, et al. An inflammatory cytokine signature predicts COVID-19 severity and survival. Nat Med 2020; 26: 1636-43.

18. Ragab D, Salah Eldin H, Taeimah M, et al. The COVID-19 cytokine storm: what we know so far. Front Immunol 2020; 11 1446.

19. Zuo T, Zhang F, Lui GCY, et al. Alterations in gut microbiota of patients with COVID-19 during time of hospitalization. Gastroenterology 2020; 159: 944-55e948.
20. Zielecki P, Kaniewska M, Furmanek M, et al. Effective treatment of severe acute pancreatitis and COVID-19 pneumonia with tocilizumab. Gastroenterology Rev 2020; 15: 267-72.

21. Group RC, Horby P, Lim WS, et al. Dexamethasone in hospitalized patients with Covid-19. N Engl J Med 2021; 384: 693-704.

22. COVID-19 Treatment Guidelines Panel. Coronavirus Disease 2019 (COVID-19) Treatment Guidelines. National Institutes of Health. Available at https://www.covid19treatmentguidelines. nih.gov/. Accessed August 4, 2021.

23. Cox MJ, Loman N, Bogaert D, et al. Co-infections: potentially lethal and unexplored in COVID-19. Lancet Microbe 2020; 1: e11.

24. Kukla M, Adrych K, Dobrowolska A, et al. Guidelines for Clostridium difficile infection in adults. Gastroenterology Rev 2020; 15: 1-21.

25. Lewandowski K, Rosolowski M, Kaniewska M, et al. Clostridioides difficile infection in coronavirus disease 2019 (COVID-19): an underestimated problem? Pol Arch Intern Med 2021; 131 : 121-7.

26. Leung C. Clinical features of deaths in the novel coronavirus epidemic in China. Rev Med Virol 2020; 30: e2103.

27. Papaefthymiou A, Koffas A, Kountouras J, et al. The impact of COVID-19 pandemic on gastrointestinal diseases: a single-center cross-sectional study in central Greece. Ann Gastroenterol 2021; 34: 323-30.

28. Livanos AE, Jha D, Cossarini F, et al. Gastrointestinal involvement attenuates COVID-19 severity and mortality. medRxiv 2020. doi: https://doi.org/10.1101/2020.09.07.20187666.

29. Pan L, Mu M, Yang P, et al. Clinical characteristics of COVID-19 patients with digestive symptoms in Hubei, China: a descriptive, cross-sectional, multicenter study. Am J Gastroenterol 2020; 115: 766-73.

30. Baradaran A, Ebrahimzadeh $\mathrm{MH}$, Baradaran A, et al. Prevalence of comorbidities in COVID-19 patients: a systematic review and meta-analysis. Arch Bone Jt Surg 2020; 8: 247-55.

31. Hojyo S, Uchida M, Tanaka K, et al. How COVID-19 induces cytokine storm with high mortality. Inflamm Regen 2020; 40: 37.

32. D’Amico F, Baumgart DC, Danese S, et al. Diarrhea during COVID-19 infection: pathogenesis, epidemiology, prevention, and management. Clin Gastroenterol Hepatol 2020; 18: 166372.

Received: 20.08.2021

Accepted: 29.08 .2021 